\title{
Videos in the medical student objective structured clinical examination: A systematic review
}

\author{
H-D. Pham ${ }^{1}$, E. B. Pistorius' ${ }^{1}$, S. Bacchi ${ }^{2}$, H. P. A. Jersmann ${ }^{1}$
}

\begin{abstract}
Introduction: The use of video in objective structured clinical examinations (OSCEs) may serve as a means of facilitating assessment, as a component of the exam itself or utilised as a tool in other ways. The aim of this review was to summarise the evidence currently supporting the uses of video in relation to the OSCE process.

Methods: A systematic search of Pubmed, Medline, EMBASE, Scopus and PsychInfo was conducted on 12 April 2017 using the subject heading "(OSCE OR objective structured clinical examination) AND (video OR recording)". To be deemed eligible, the paper had to be a primary research paper, involve the analysis of OSCEs conducted for medical students and involve the use of video technology.

Results: Thirty-six articles met eligibility criteria. Twenty-four investigated the use of video within OSCEs as a means of facilitating the exam, nine detailed cases where video was integrated as a part of an OSCE and three utilised videos in some other way. Of those that investigated the use of video as a means of facilitation, only one compared the use of video to traditional in-room examiners. Other articles in this category demonstrated good inter-rater reliability between different assessors marking via video.

Conclusion: There is currently limited evidence regarding the usefulness and educational benefit of introducing video into the assessment of clinical skills, and minimal research evaluating the use of video compared to traditional live examiners as a means of facilitating the assessment of students currently exists. Preliminary results demonstrated reasonably high inter-rater reliability. This should be a topic of future research.
\end{abstract}

Keywords: education, medical; educational measurement; students, medical; medical students; videotape recording; video recording; assessment; education; evaluation.

1 University of Adelaide

2 Queen Elizabeth Hospital

\section{Correspondence}

Huy-Dat Pham

University of Adelaide

4 North Terrace

Adelaide, SA 5005

Australia

Phone: +61 435268627

Email: huy-dat.pham@student.adelaide.edu.au 


\section{Introduction}

Objective structured clinical examinations (OSCEs) have long been used in the assessment of medical students' communication skills, clinical skills (including physical examination and history taking) and management of patients. Video has had a role in the OSCE process in the past and may play a larger role in the future because of a variety of benefits, including but not limited to easing the burden on examiners who may have time constraints or other commitments, decreasing logistical burden of coordinating multiple examiners to assess in-person and allowing for greater quality control and objectivity (Troncon, 2004; Vivekananda-Schmidt et al., 2007).

Video has been implemented in OSCEs in a variety of manners. For example, video has had a role in some OSCEs as a means to deliver a question stem or other scenario-based content (e.g., video of a patient demonstrating a particular sign). This use of video in an OSCE is usually termed an objective structured video examination (OSVE). Video has also been used to record OSCE stations for later student review. In addition, it has had a role in evaluating components of OSCEs. While video has been used in all of these manners, current evidence supporting their use is inconclusive.

This paper evaluates current literature published in peer-reviewed journals that reports on evidence regarding the use of video employed in relation to any form of evaluation in the OSCE process.

\section{Method}

The preferred reporting items for systematic review and meta-analysis protocols (PRISMA-P) guidelines were used to develop the method for this systematic review (Shamseer et al., 2015). The databases Pubmed, Medline, EMBASE, Scopus and PsychInfo were searched using combinations of the subject headings: "(OSCE OR objective structured clinical examination) AND (video OR recording)". The original online searches were conducted on 12 April 2017. Articles were then restricted to those published in English.

Following the application of this filter, titles and abstracts of the remaining publications were viewed to determine if they met eligibility criteria. To be eligible for inclusion, a study had to: (i) be a primary research paper that includes a control group (for example, not a case report, case series, editorial or review), (ii) involve the analysis of OSCEs conducted for undergraduate or graduate medical students (for example, not assessed for nursing, other allied health faculties or post-graduate OSCEs) and (iii) involve the use of video technology at any stage during the OSCE (for example, studies were not used if the video was included as a teaching tool prior to assessment or as a tool for training staff and was, therefore, separate from the assessment of medical students). Three ways in which video may be used during an OSCE were defined: (A) as a means of facilitating assessment, (B) delivering OSCE content or as a component of a station and (C) any other function relating to the OSCE not previously defined. 
For this review, nursing, other allied health faculties and post-graduate OSCEs were not included to avoid inconsistencies in results that may be due to differences in OSCE format, assessment and conventions between different disciplines.

Articles that appeared likely to fit eligibility criteria were then reviewed in full text. If it could not be determined whether an article met eligibility criteria, it was retrieved and viewed in full text prior to inclusion or exclusion. All reference lists of included articles were then reviewed for further studies that might fit the inclusion criteria. Data extraction and quality analysis of the included articles was performed by the two student authors, using a standardised form. Aspects of studies that were examined to evaluate quality were adapted from the Medical Education Research Study Quality Instrument (D. Reed et al., 2007). These elements of studies included study design (in particular whether there was a single group or multiple groups, and if there was randomisation), the number of institutions involved, sample size, reporting of response rate, the type of data collected and the nature of the outcomes that were measured. However, no formal quality scores were calculated. Disagreements with regards to data extraction and quality analysis were resolved by discussion between the authors $\mathrm{HP}$ and $\mathrm{EP}$ until consensus was reached. If consensus was not reached, the issue was discussed with a third author (SB).

\section{Results}

The initial search identified 775 potential articles; 745 of these articles were published in English. After reviewing the titles and abstracts of these articles, 173 were reviewed in full text. Thirty-six articles were found to meet the inclusion/exclusion criteria and were included in the review (see Figure 1)

A large number of articles ( 83 in total) were deemed inappropriate to be included in this review, as they specifically referred to OSCEs used in other health professions, such as nursing, other allied health fields and postgraduate training in various fields. A followup review may be warranted to assess the scope of video use in OSCEs across all health professions.

The 36 included articles were subsequently divided into one of three categories, based on the context of the use of videos within the medical student OSCE/s. These categories were:
A. means of facilitating assessment
B. a component of station or OSCE content
C. any other function related to OSCE.

Following this, 24 articles were deemed to fit in Category A, nine in Category B and three in Category C. 


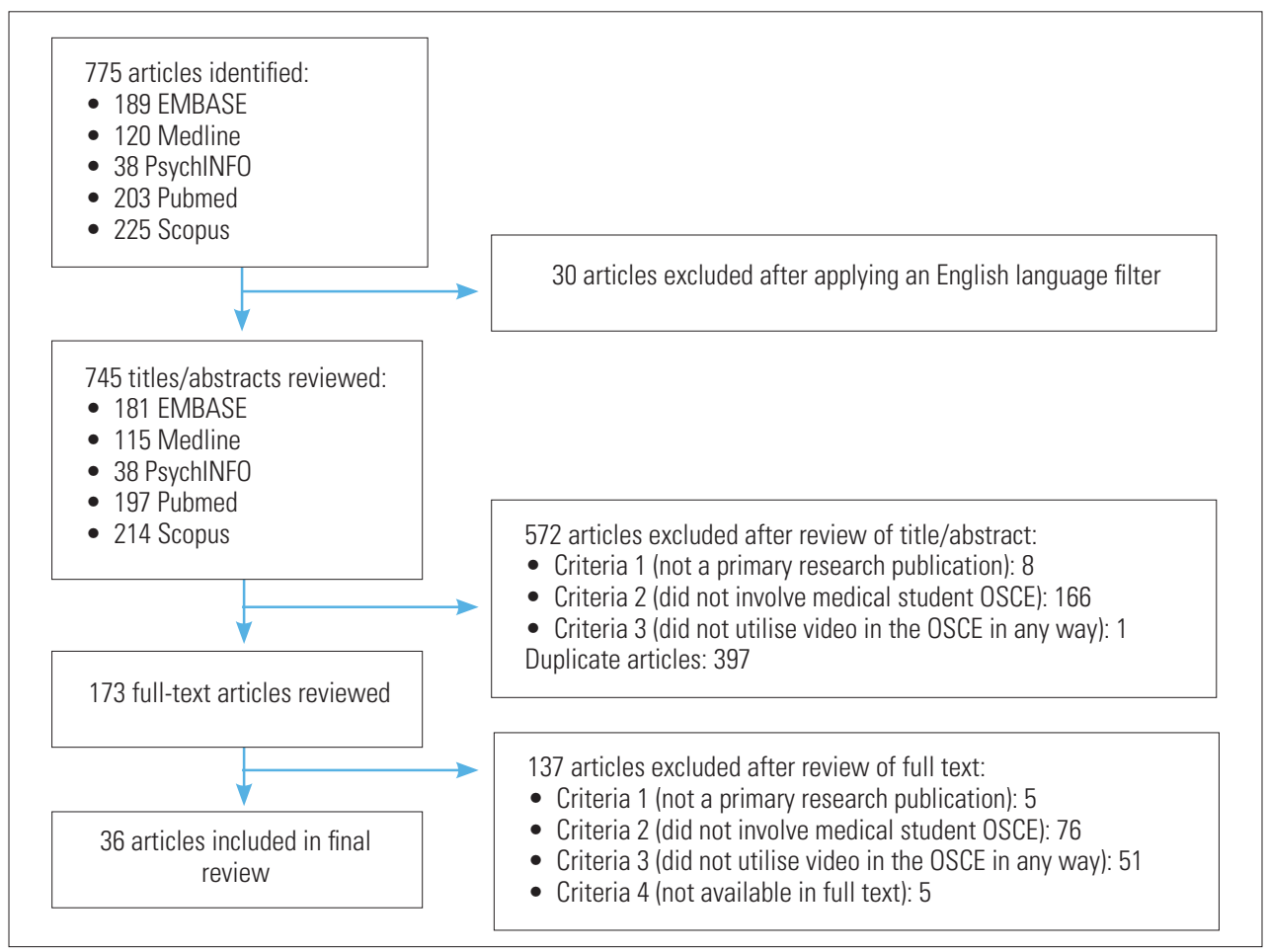

Figure 1. Flowchart detailing results from the search strategy and application of inclusion and exclusion criteria for a review of articles that discussed the use of video in objective structured clinical examinations.

\section{Prevalence of video-recorded OSCE assessment}

Out of the original 36 articles, all articles within Category A reported on video being used for assessment, record-keeping or analysis. Within Category A, eight articles directly analysed the use of video to record students' OSCEs for later evaluation and marking (e.g., in order to compare video versus live assessor marking or to solely mark via video assessor), while 16 articles reported on video being utilised to analyse a component of the OSCE process that is not related to the student's academic performance. In addition, three instances of video recording assessments were noted in Category C. This totals 27 articles. One article within Category B reported video-recording of the OSCEs in any manner (Walters, Osborn, \& Raven, 2005).

Black and Harden (1986) was noted as the oldest recorded reference to video recording in an OSCE. This prospective study of 37 undergraduate medical students trialled video recordings as a means of feedback for students and was compared to other methods of feedback. Black and Harden found that checklist plus videotape part way through the examination was the most preferred method of feedback. 


\section{Category A: Means of facilitating assessment}

\section{Assessment of video recording as a means of assessment against traditional (non-video) methods}

Only one study, conducted by Vivekanada-Schmidt et al. (2007), addressed and compared any component of video recording as a means of assessment against traditional methods (live marking by an examiner). It was undertaken in the United Kingdom on 95 thirdyear undergraduate medical students. Vivekanada-Schmidt et al. specifically studied the reliability within and between assessment methods of OSCEs involving joint examination of the shoulder and knee and found that the video assessor consistently scored students lower using the Global Rating Scale (GRS) score. In addition, analysis of the mean scores for checklist assessment showed lower mean scores by the video assessor compared to the live assessor. Intraclass correlation showed moderate reliability between live and video assessors for both the checklist score and the GRS score. Finally, statistical agreement was shown to be $85 \%$ between live and video assessors for the shoulder examination and $87 \%$ for the knee examination.

\section{Inter-rater reliability}

There was a total of six articles out of the final 36 that analysed inter-rater reliability in some manner. Five of these articles analysed inter-rater reliability where all scores were given by video assessors (Han et al., 2016; Huntley, Salmon, Fisher, Fletcher, \& Young, 2012; Ishikawa, Hashimoto, Kinoshita, \& Yano, 2010; Kiehl et al., 2014; V. A. Reed, 1998). One article analysed reliability between video and live assessors (VivekanandaSchmidt et al., 2007).

From the five articles analysing inter-rater reliability where all scores were given by video assessors, all five articles found and demonstrated almost identical results. Each article assessed reliability using intraclass correlation, which describes how strongly individual scores from assessors resembled others within the same group, and found intraclass correlation coefficients (ICC) ranging from 0.73 (Huntley et al., 2012; Ishikawa et al., 2010) to 0.79 (Han et al., 2016), indicating a range from the high end of "good" reliability to the low end of "excellent" reliability. The exception to this was V. A. Reed's (1998) analysis of inter-rater agreement as part of a 1997 analysis of OSCEs as a means of assessment for the, then novel, patient video interview (PVI). V. A. Reed used the analysis technique of canonical correlation to compare agreement. Her results showed that out of the 33 students analysed, there was an $86.7 \%$ absolute agreement of score, however correlation was $r=-0.62$, which demonstrates a strong negative correlation.

Kiehl et al. (2014) specifically developed and assessed a method of standardised and quality-assured assessment in undergraduate medicine. One hundred and fifty-five fifthyear undergraduate medical students participated in this study. A final 78 videos were collected and analysed for inter-rater reliability between two independent examiners assessing three informed consent scenarios via standardised checklist. The 155 students formed pairs to prepare for and undergo the examination. Students took turns to perform the examination and to film the interaction. Then, they selected one video recording out 
of the two filmed to submit for analysis by the independent examiners, which explains the reason for 78 videos instead of 155 . It is unclear why this decision was made. The results showed an ICC of 0.83 (95\% CI: $0.66-0.92, p<0.001)$ for the appendectomy scenario, 0.73 (95\% CI: $0.46-0.87, p<0.001)$ for the cholecystectomy scenario and $0.76(95 \%$ CI: $0.54-0.89, p<0.001)$ for the hernia repair scenario. This resulted in a mean ICC of 0.78 (95\% CI: $0.67-0.85, p<0.001$ ), indicating "excellent" reliability between assessors (Kiehl et al., 2014).

Another key study supporting this finding was Han et al. (2016), where six independent video assessors marked 52 encounters using a newly developed 4-item checklist-type measure of assessment named the BRISK (brief risk information skill) scale. In this study, inter-rater reliability between the six assessors was once again high, showing an ICC of 0.79 (95\% CI: $0.71-0.81, p<0.001$ ) for consistency and an ICC of 0.73 (95\% CI: 0.65$0.88, p<0.001)$ for absolute agreement, indicating "excellent" and "good" reliability, respectively. Han et al. also calculated ICCs for all pairs of assessors. The lowest ICC for consistency (0.50) was found between raters 1 and 4, indicating "fair" reliability, and the lowest ICC of absolute agreement (0.46) was found between raters 2 and 4, once again indicating "fair" reliability. The highest ICC for consistency (0.92) was found between raters 3 and 6 and highest ICC for absolute agreement $(0.90)$ was found between raters 3 and 5, both indicating "excellent" reliability (Han et al., 2016).

\section{Category B: A component of station or OSCE content}

\section{Use of video as a component of the OSCE}

When analysing articles citing video for OSCE use, there were two distinct sub-categories found. The first was instances where video was used as an aid or scenario integrated with the action of physical examination, history taking or another clinical skill. The second was instances where video scenarios were presented, followed by questions, answered orally or written, that did not strictly involve performing a physical examination, history or another clinical skill. For the purpose of this review, the first sub-category will be termed video objective structured clinical examination (VOSCE) and the second sub-category will be termed objective structured video examination (OSVE), as is cited in some of the articles involved (Hulsman, Mollema, Hoos, de Haes, \& Donnison-Speijer, 2004; Hulsman, Mollema, Oort, Hoos, \& de Haes, 2006; Watson, Stevenson, \& Hawkins, 2016). In total, two articles were found to report on VOSCEs.

In 2002, Malloy investigated the effectiveness of enhanced web-learning modules for otitis media cases assessed by history-taking, diagnosis and counselling OSCE stations, where part of the stimulus was presented via video. Specifically, the video showed a pneumoscopic examination to support the history findings.

In 2005, Walters et al. (2005) incorporated video into both the scenario and assessment. As part of a 17-18 station OSCE circuit, two stations featured a 4-minute video scenario preceding the medical students recording a mental-state examination. Another station featured a video vignette followed by oral problem-oriented questions. Therefore, Walters et al.'s OSCE circuit demonstrated instances of both VOSCEs and OSVEs. 
The remaining seven articles within Category B, as well as the study by Walters et al. (2005), demonstrated and analysed the use of OSVEs.

Hulsman et al. (2004) and Hulsman et al. (2006) conducted studies of OSVEs. In both cases, they featured short video scenarios followed by short-essay questions. The study reported in 2006 featured three video cases of history taking, breaking bad news and decision making. Two hundred medical students were involved, and a total of 116 completed all three scenarios.

\section{Evaluation of VOSCEs and OSVES via subjective survey}

Five articles were identified that assessed and reported on the effectiveness of VOSCE or OSVE via a subjective survey completed by students and/or examiners (Hulsman et al., 2004; Sulaiman \& Hamdy, 2013; Vlantis, Lee, \& Van Hasselt, 2004; Walters et al., 2005; Watson et al., 2016). All studies found unanimous support and positive feedback for the use of videos either within VOSCEs or OSVEs.

For example, Walters et al. (2005) surveyed a total of 128 students, who compared the use of VOSCE to OSVE and to traditional OSCE stations. The researchers found that "most students expressed a preference for interactive and video stations over written stations [and that] time constraints at various stations were often raised as a cause for concern, in particular for written and video stations" (p. 295).

Another key study to support the use of videos in OSCEs was conducted by Vlantis et al. (2004) on 161 sixth-year undergraduate medical students from China. Surveys showed $68.7 \%$ of students perceived the OSVE to be a fair exam of their practical skills and $80 \%$ of students perceived the OSVE to be a fair exam of their ear, nose and throat (ENT) knowledge (Vlantis et al., 2004).

\section{Evaluation of VOSCEs and OSVEs via statistical comparison}

Six articles were identified that assessed and reported on the effectiveness of VOSCE or OSVE via statistical comparison of the VOSCE/OSVE (Hulsman et al., 2006; Humphris \& Kaney, 2000; Malloy, 2002; McWilliam, Davidson, \& Pugh, 2009; Sulaiman \& Hamdy, 2013; Walters et al., 2005). Out of these six studies, one did not directly address the OSVE (Malloy, 2002); one did not compare results to other sources (McWilliam et al., 2009); one compared student results to consultant attachment grades (Walters et al., 2005); one analysed reliability of assessment only within the scope of the OSVE (Hulsman et al., 2006); and finally, two studies compared student performance in the OSVE with other assessment methods (Humphris \& Kaney, 2000; Sulaiman \& Hamdy, 2013).

These six articles within this category differed in their methods and statistical analysis. However, despite this heterogeneity, the majority of results concluded that the use of videos in OSCEs is warranted.

For example, Sulaiman et al. (2013) compared OSVE (named clinical image and video assessment (CIVA)) results to OSCE grades, multiple-choice question (MCQ) grades, direct observation clinical encounter exam (DOCEE) grades and written exam scores 
using the 2-sided Pearson correlation analysis with reliability analysed using Cronbach's alpha coefficient. The strongest correlation was discovered between the OSVE (CIVA) and OSCE grades $(r=0.83, p<0.001)$. This is converse to comparison with MCQ, DOCEE and written exam scores, which all demonstrated poor correlation. There was good reliability between OSCE scores and CIVA scores, with respective scores of 0.71 and 0.78 for the first batch of students and 0.91 and 0.91 for the second batch.

Another key study supporting this finding was Humphris et al. (2000). This study compared the performance of 200 students in the OSVE against the OSCE, using the Global Simulated Patient Rating Scale (GSPRS) and the Liverpool Communication Skills Assessment Scale, which was marked by expert examiners. The OSVE rating appeared to be independent from the simulated patient global ratings $(r=0.026$; CI: $-0.052,0.105$; $p=0.60)$ but positively and significantly related to the examiner ratings $(r=0.173, \mathrm{CI}$ : $0.095,0.249 ; p=0.0001)$. The relationship between the simulated patient and expert examiner ratings was strong $(r=0.638$, CI: $0.588,0.682 ; p=0.0001)$.

\section{Evaluation of VOSCEs and OSVEs checklist style questionnaire}

One study assessed reception of VOSCE/OSVE via a checklist-style questionnaire. Hulsman et al. (2004) gathered data on student perception of the OSVE via a 10-question survey. Nine of the questions used 3- or 4-point Likert scales. Results showed that the majority of students $(68.2 \%)$ considered the entire assessment to be "very good" or "good" for the assessment of communication skills.

\section{Discussion}

The heterogeneity of the 36 articles meant that, following analysis, the primary and most pressing finding was the limited literature available regarding each of the various specified uses of video in objective structured clinical examinations for medical students. This was true for all variants of video use, e.g., assessment or conducting the exam.

Analysis of the available literature shows promise for the use of videos in OSCEs and its efficacy as a tool in assessment, with the vast majority of current research indicating good reliability between assessors in varying contexts. There is also sufficient evidence to suggest students and assessors benefit from videos in both VOSCE and OSVE formats.

From the studies analysed, there is clearly scope to further study the differences and reliability between video-recorded and live-assessed OSCEs. The potential benefits of videorecorded assessment are: easily accessible moderation of results, greater standardisation of assessment and standardised environments for the student. The analysed studies demonstrated a high inter-rater reliability for video-recorded assessment, however future comparison of these results to live-assessed OSCEs is necessary for sound conclusions to be made in addition to the one article currently published. It is also noted that this single article only analysed a musculoskeletal exam. It is reasonable to hypothesise that there may be significant differences with examinations requiring greater student-patient communications, such as a psychiatric interview or counselling station. 
Another key area for further research is in the development, testing and analysis of video objective structured clinical examinations (VOSCEs). There is a moderate amount of research conducted on OSVEs where clinical skills are not assessed in conjunction with video usage, however very limited research has been conducted on the usefulness and educational benefit of introducing video into the assessment of clinical skills.

The studies identified in this review have several limitations. The majority of studies included in Category A did not compare video assessment to live assessment and, therefore, did not give a reliable determination of video assessment reliability. Furthermore, the majority of studies in Category B did not investigate the use of videos in a context where students conducted clinical tasks. The implication of this is a lack of literature to address the further development and implementation of new OSCE formats. Finally, no studies in Category B compared the students' results in VOSCEs or OSVEs to a gold standard of assessment; therefore, it is difficult to conclude how accurate VOSCEs or OSVEs are as a representation of student ability.

As previously mentioned, during the eligibility determination stage of this review, a total of 89 papers were excluded because they assessed OSCEs conducted for other health professional students or in postgraduate contexts. It is entirely plausible, and likely, that combining the findings of this review with the excluded papers would yield some insights absent from the limited literature available purely for medical students, especially in an era where interprofessional learning and collaborative health professional education is becoming more commonplace. Furthermore, this review is limited to only video OSCEs. The implication of this is that more detailed information and comparison may be possible if studies of traditional OSCEs were compared to findings from this review; in particular, statistical comparison of inter-rater reliability in each context would be useful. This review also has several other limitations that need to be acknowledged. The exclusion of articles not published in English may have discounted relevant studies. Similarly, there were five articles that could not be retrieved in full text. With a review of this nature, there is also the possibility of publication bias affecting results, e.g., studies reporting significant outcomes being more likely to be published.

Finally, when considering VOSCEs as a replacement for in-room examiners, it is important to note that there may be many potential downfalls not addressed in current literature. For example, if the VOSCE (without in-room examiner) was to be used in a summative manner, management of technical problems would pose significant logistical issues to the administering academic institution. In light of this, we suggest that in the design of VOSCEs, it might be wise to include multiple redundancies and safeguards to mitigate this risk. However, further research may be required to justify this suggestion.

\section{Conclusion}

The various uses of video recordings in OSCEs are well established. Video has been used for evaluating components of OSCEs, student performance and other factors; as a 
component of an OSCE itself; and for various other purposes discussed above. Analysis of the studies that discuss inter-rater reliability has shown overall high inter-rater reliability for the majority of studies conducted thus far. Additionally, studies showed approval from students, who provided support and positive feedback for the use of videos either within VOSCEs or OSVEs. What is lacking, however, is literature evaluating the use of video compared to traditional live examiners as a means of facilitating the assessment of students. Only one such paper was found to analyse this. Given the above findings, there is significant scope and benefit for future investigation of the logistical and educational advantages of introducing video into the assessment of clinical skills.

\section{Funding and conflict of interest}

None

\section{References}

Black, N. M. I., \& Harden, R. M. (1986). Providing feedback to students on clinical skills by using the objective structured clinical examination. Medical Education, 20(1), 48-52. doi:10.1111/j.1365-2923.1986.tb01041.x

Han, P. K., Joekes, K., Mills, G., Gutheil, C., Smith, K., Cochran, N. E., \& Elwyn, G. (2016). Development and evaluation of the "BRISK Scale," a brief observational measure of risk communication competence. Patient Education and Counseling, 99(12), 2091-2094. doi:10.1016/j.pec.2016.08.013

Hulsman, R. L., Mollema, E. D., Hoos, A. M., de Haes, J. C., \& DonnisonSpeijer, J. D. (2004). Assessment of medical communication skills by computer: Assessment method and student experiences. Medical Education, 38(8), 813-824. doi:10.1111/j.1365-2929.2004.01900.x

Hulsman, R. L., Mollema, E. D., Oort, F. J., Hoos, A. M., \& de Haes, J. C. (2006). Using standardized video cases for assessment of medical communication skills: Reliability of an objective structured video examination by computer. Patient Education and Counseling, 60(1), 24-31. doi:10.1016/j.pec.2004.11.010

Humphris, G. M., \& Kaney, S. (2000). The objective structured video exam for assessment of communication skills. Medical Education, 34(11), 939-945. doi:10.1046/j.1365-2923.2000.00792.x

Huntley, C. D., Salmon, P., Fisher, P. L., Fletcher, I., \& Young, B. (2012). LUCAS: A theoretically informed instrument to assess clinical communication in objective structured clinical examinations. Medical Education, 46(3), 267-276. doi:10.1111/j.1365-2923.2011.04162.x

Ishikawa, H., Hashimoto, H., Kinoshita, M., \& Yano, E. (2010). Can nonverbal communication skills be taught? Medical Teacher, 32(10), 860-863. doi:10.3109/01421591003728211 
Kiehl, C., Simmenroth-Nayda, A., Goerlich, Y., Entwistle, A., Schiekirka, S., Ghadimi, B. M., . . . Koenig, S. (2014). Standardized and quality-assured video recorded examination in undergraduate education: Informed consent prior to surgery. Journal of Surgical Research, 191(1), 64-73. doi:10.1016/j.jss.2014.01.048

Malloy, M. H. (2002). Enhanced web-based otitis study case vs simple papercase: Impact on medical student objective structured clinical exam (OSCE) performance. Medical Education, 7(1), 4532. doi:10.3402/meo.v7i.4532

McWilliam, C., Davidson, I., \& Pugh, A. (2009). Experience in use of audio-visual examinations in psychiatry for final M.B. Ch. B. Medical Teacher, 8(4), 373-375. doi: $10.3109 / 01421598609028996$

Reed, D., Cook, D. A., Beckman, T., Levine, R., Kern, D., \& Wright, S. (2007). Association between funding and quality of published medical education research. JAMA, 298(9), 1002-1009.

Reed, V. A. (1998). Assessing communication skills in medical education: Development of the patient video interview. Retrieved from http://libarchive.dartmouth.edu/cdm/ ref/collection/dcdis/id/180420 .

Shamseer, L., Moher, D., Clarke, M., Ghersi, D., Liberati, A., Petticrew, M., . . . Group, P.-P. (2015). Preferred reporting items for systematic review and metaanalysis protocols (PRISMA-P) 2015: Elaboration and explanation. BMJ, 350, g7647. doi:10.1136/bmj.g7647

Sulaiman, N. D., \& Hamdy, H. (2013). Assessment of clinical competencies using clinical images and videos"CIVA". BMC Medical Education, 13(1), 78. doi:10.1186/1472-6920-13-78

Troncon, L. E. D. A. (2004). Clinical skills assessment: Limitations to the introduction of an "OSCE" (objective structured clinical examination) in a traditional Brazilian medical school. Sao Paulo Medical Journal, 122(1), 12-17.

Vivekananda-Schmidt, P., Lewis, M., Coady, D., Morley, C., Kay, L., Walker, D., \& Hassell, A. B. (2007). Exploring the use of videotaped objective structured clinical examination in the assessment of joint examination skills of medical students. Arthritis \& Rheumatism, 57(5), 869-876. doi:10.1002/art.22763

Vlantis, A. C., Lee, W. C., \& Van Hasselt, C. A. (2004). The objective structured video examination of medical students. Medical Education, 38(11), 1199-1200. doi:10.1111/j.1365-2929.2004.01995.x

Walters, K., Osborn, D., \& Raven, P. (2005). The development, validity and reliability of a multimodality objective structured clinical examination in psychiatry. Medical Education, 39(3), 292-298. doi:10.1111/j.13652929.2005.02091.x

Watson, P., Stevenson, M., \& Hawkins, S. (2016). Neurology assessment by objective structured video examination. The Clinical Teacher, 13(5), 348-351. doi:10.1111/ tct. 12443 